\title{
Challenges and Solutions of Applying Large-Scale Agile at Organizational Level
}

\author{
Florin DUMITRIU, Gabriela MEȘNIȚĂ, Laura-Diana RADU \\ Department of Business Information Systems, Faculty of Economics and Business Admin- \\ istration, Alexandru Ioan Cuza University of Iasi \\ fdumi@uaic.ro,gabriela.mesnita@feaa.uaic.ro,glaura@uaic.ro
}

Large-scale agile development receives widespread interest today in research and practice. Two different dimensions could be considered in the context of applying agile at large-scale: individual level, like project or team, and organization level. The majority of literature treats the subject of large-scale agile implementation at the individual level or not very specific. In our paper, we placed at organization level with the aim to find out the challenges and solutions that are relevant for enterprise-wide agile implementation in large organizations in order to create agility beyond individual team or project. Based on the literature review, we have found twelve challenges, grouped in three categories and three general solutions.

Keywords: Large-Scale Agile, Organizational Context, Hybrid Method, Enterprise Architecture

1 Introduction

Over the past 15 years, agile software development has increasingly become the norm in the IT industry. Many organizations adopt agile development to become more competitive by improving the software quality, reducing costs and time to market and managing changing requirements. Even though the initial fundamental assumptions underpinning agile approach suggested that it is best suited to small projects and single, small development teams, the agile methods are increasingly applied in different contexts, such as distributed development settings, high-integrity software projects and at large-scale, in projects with tens of teams and hundreds of developers [1]. The implementation of agile methods in these contexts encounters various challenges.

In the field of applying agile at large-scale, a clear classification of the existing streams of research is difficult to recognize [2] due to the lack of general agreement to the actual definition of "large-scale". Various expressions have been used in the literature to denote the "large-scale" term. Some researchers and practitioners have used general terms, like "large-scale settings" or "large contexts", while others have employed one or even many more specific phrases such "large projects", "large teams", "large, complex software", "large organization" or "mature organization" [3]. Another definition perceives the "largeness" in terms of the complexity of the various knowledge boundaries across the actors and technologies involved [4].

If we look at all those phrases, it can be easily noticed that two different dimensions could be considered for the term "large-scale" in the context of applying agile methodology: individual level, like project or team, and organizational level, often referred by large or mature organization. The two dimensions of applying agile approach should be treated separately as the challenges, success factors and solutions are different.

The majority of literature treats the subject of large-scale agile implementation at the individual level or not very specific. Only a few papers have explicitly assumed an organization perspective when analyzing agile adoption at large [5], [6], [7]. Also, through our literature review, we have found that some papers mention that they deal with agile issues in large organization, but their approach and results are focused on agile for large software project or large-scale agile development [8] or a mixed of those with large organization perspective [6].

In our paper we are adopting an organizational level perspective with the aim to analyze how 
agile development methodologies can be applied in large companies to best fit to an organizational context. Our objective is to investigate the literature on the field of applying agile at large-scale in order to find out the challenges and solutions that are relevant for applying large-scale agile at organizational level with the aim to create agility beyond individual team/project level.

The rest of the paper is organized as follows. In Section 2, we present the research method. In Section 3, we discuss the literature on the topic of company-wide adoption of agile methods with the aim of identifying the challenges and solutions, while in Discussion Section we analyze in more details the main challenges and related solutions proposed in the literature. Finally, Section 5 concludes the article.

\section{Research Methodology}

In this paper, we present the results of our literature review on the topic of applying agile at large-scale in an organizational context. Our research questions are:

RQ1: What are the challenges that are relevant for applying large-scale agile at the organization level and how could they be grouped for a better understanding?

RQ2: What solutions can be used for applying large-scale agile in organizational context?

The research process consisted of four main steps. In the first step, we searched in several academic databases and search engines, such as AIS Electronic Library (AISeL), ACM Digital Library, Web of Science, and Science Direct. Addressing our research questions, we selected a list of papers that contain in their title relevant expressions for large-scale agile, such as "agile in large settings", "large-scale agile", "agile at large", "scaling agile". We used EndNote to exclude redundant articles, conference reviews, and announcements of conferences or other events. We identified 312 papers focused on the topic. In the next step we have excluded those papers that contained in their title's relevant expressions for individual levels, like "team", "project", or "software". For the rest of the papers we read keywords and abstracts, and eliminated the papers about organizational agility with a managerial perspective, and those on applying agile in distributed environments as they were focused on specific issues for geographically distributed teams. This process led to a final number of 63 articles that we considered for further analysis. In the last step, we deeply analyzed the remaining papers to extract the relevant ideas for applying agile in large organizations. In this respect we considered issues like "multi-team environment", "enterprise architecture", and "organizational change".

\section{Literature Review}

In order to address our research questions, we have conducted a literature review with the aim of identifying the challenges and solutions for adopting agile in an organizational context.

In this section we present our findings. First, we present the challenges identified and the categories used for grouping them to bring a better understanding. In the next subsection the solution for adopting agile at the organization level are described.

\subsection{Challenges of Applying Large-Scale Ag- ile In Organizational Context}

Organizations try to adopt agile practices to become more competitive, improve processes in order to manage changing requirements, but they face additional challenges in the integration of agile development at the organizational level. Although many companies use agile methods, it is still unclear in which environments and under what conditions they really work [9]. Some researchers have stated that agile development in its pure form is likely not a good solution for large, traditional systems development organizations [10], [11]. The number and the classifications of challenges vary from article to article, depending on the research methodology used, the goal of the article and the time in which the research was conducted. The complexity of the subject results from the 79 challenges grouped into 11 categories identified by [12]. On the other side, [13] use two broad groups of challenges. First category includes challenges related to the methods themselves, "because of the fixed 
rule bases and assumptions built into the methods". The second category consists in challenges induced by the enterprise that "will prevent the successful application of the new methods".
We have focused to those challenges of agile adoption that arose at the organizational level in large organizations. The main challenges and their grouping are outlined in Table 1.

Table 1. Challenges of agile adoption in large organizations

\begin{tabular}{|c|c|c|}
\hline Content & Details & Source \\
\hline \multirow{4}{*}{$\begin{array}{l}\text { Multi- } \\
\text { team/multi-pro- } \\
\text { ject environ- } \\
\text { ment }\end{array}$} & Implementing self-organizing teams. & {$[4],[6],[13],[10]$} \\
\hline & Coordination of several agile teams. & {$[4],[6],[10],[11]$} \\
\hline & $\begin{array}{l}\text { Different interpretations of agile principles and prac- } \\
\text { tices among agile teams. }\end{array}$ & [14] \\
\hline & Managing and sharing knowledge with stakeholders. & [12] \\
\hline \multirow{5}{*}{$\begin{array}{l}\text { Organizational } \\
\text { transfor- } \\
\text { mation/change }\end{array}$} & Management change. & {$[6],[11]$} \\
\hline & Culture and leadership behavior change. & [8] \\
\hline & Specialized knowledge in silos. & {$[6],[11]$} \\
\hline & Integrating non-development functions. & {$[6],[11]$} \\
\hline & $\begin{array}{l}\text { Integration of agile projects with the existing software } \\
\text { development processes. }\end{array}$ & [9], [13] \\
\hline \multirow{3}{*}{$\begin{array}{l}\text { Alignment of in- } \\
\text { dividual projects } \\
\text { to enterprise } \\
\text { business goals }\end{array}$} & $\begin{array}{l}\text { Conflicts between agile projects and holistic enterprise } \\
\text { architecture (EA). }\end{array}$ & {$[10],[15]$} \\
\hline & Integration of EA frameworks and agile development. & [15], [16] \\
\hline & $\begin{array}{l}\text { Balancing between the agility of individual projects } \\
\text { and the organization agility. }\end{array}$ & {$[17]$} \\
\hline
\end{tabular}

The first category includes the challenges that arise in multi-team/multi-project environment specific for large organizations. One of the most prominent challenges for applying agile at large is the difficulty in coordinating the work of several agile teams [4], [10], [11]. Large organizations naturally have many interdependencies which require a large amount of coordination that could be hard to manage through mutual adjustment as in agile methods. The team is just one step in an organization's value creation process, and each team has to coordinate their work with other teams. It is quite difficult to bring agility beyond the team as the introduction of agile might create flexibility at the team level, but the surrounding organization could not be responsive enough [6]. Another challenge in multi-team environments is related to the problems that arise with the self-organizing team principle [4], [6], [10], [13], one of the fundamental assumptions in agile approach. Self-organizing means that a team makes decisions through informal communication and frequent, short meetings rather than relying on one owner to guide the project.

Large organizations involve numerous people in many development teams. This situation may lead to a lack of alignment between teams regarding the use and implementation of agile practices [14]. Another issue related to multiteam environment is the management and sharing knowledge with stakeholders; there are some diverging views of knowledge sharing and transfer on agile projects because while agile teams spend time to transfer information internally, they share little information with external groups [12]. Agile methods have created ways to transfer and share knowledge within the team, but still fail to search and sharing it externally. Therefore, it is a challenge to manage knowledge about system components and their dependencies with all stakeholders involved in the wide-enterprise agile implementation. 
The second category of challenges encompasses the organization change/transformation. Dikert et al. (cited in[6]) have presented three challenges that we consider relevant for applying agile at organizational level: cultural change in organization management, particularly on the middle management level because its role is unclear in agile methods; the difficulty of relocating the people due to the existence of internal boundaries, specialized knowledge in silos, or different agile cultures from team to team; the integration of non-development functions in organizations. In the case of using agile methods, organization functions ranging from marketing and sales to human resources have to be integrated in the agile environment; otherwise this might cause serious limitations for the agile implementation. In [12] two issues of organizational changes are highlighted from the perspective of the relationship with the projects' beneficiaries - internal and external projects. The internal ones would transform the entire organization, and the external ones supposed to redefine the roles of other departments and the relationships with customers, in what might become "agile end-to-end".

Integration of agile projects with the existing software development processes is also an important challenge for large organizations according to the results of a study made by [9]. Also, some empirical studies of large-scale agile have indicated challenges related to the inability to change culture and leadership behavior [8].

The last category includes the challenges regarding the alignment of individual projects to the enterprise business goals. Especially in large organizations there is a need to add some structure in business processes and the concept of enterprise architecture (EA) is put in place. Some researches stated that the need for a holistic EA when applying agile development is often overlooked [15] and may lead to several problems such as technical debt, unnecessary re-work, inconsistent communication, locally focused isolated design [10], fragile architecture, and divergence in coding style [6]. This situation creates the challenges of integrating of EA frameworks with agile methods and of balancing between projects and organization agility [15], [16], [17].

\subsection{The Solutions for Applying Agile At Or- ganization Level}

Addressing our second research question, in this section we focused on identifying the solution investigated in literature for helping agile development methodologies to fit to an organizational context. We have identified three main categories of solutions: applying agile frameworks for large-scale, integration of agile methods with the EA frameworks and the use of hybrid agile/traditional methods. Next, various issues relating to the application of these solutions will be presented.

\section{Using the frameworks for large-scale agile}

To address large-scale transformations challenges, companies have turned to specific frameworks such as Scaled Agile Framework (SAFe), Large-Scale Scrum (LeSS), Nexus, Disciplined Agile Delivery (DAD). According to [18], some of these frameworks explain very well the basics, but the agile team members talked about misunderstanding the context and routines in the case of their application under the specific conditions of the organizations.

SAFe integrates principles, practices, and competencies for lean, agile, and DevOps. This framework is organized on four level: team, program, large solution and portfolio each of them with specific activities. All these layers are tied together and offer team and program size patterns that can be used for scaling across larger organization. [19]

LeSS is a multi-team scrum framework which can be applied to 2-8 agile teams (Basic LeSS) or more than 8 teams (LeSS Huge) each having 8-12 members that work together on one specific shared product. This framework uses Scrum principles and rules on large scale projects. In LeSS there are specified the organizational changes, but also cross-functional, cross-component, end-to-end feature teams through the exclusion of traditional team lead and project manager roles [20].

Nexus extends Scrum to guide multiple Scrum Teams. It is a framework consisting of "roles, 
events, artifacts and techniques that bind and weave together the work of approximately three to nine Scrum Teams" [21]. Since Nexus extend Scrum, it can be applied in the case of the teams that already use Scrum. The main difference between these two frameworks is the addition of an integration team that is focused on facilitating the dependencies and integration issues between the teams [22].

DAD is a hybrid lean-agile approach that extends core agile development such as Scrum, Agile Modelling, Open Unified Process, eXtreme Programming, Kanban, Test-driven development and Lean. This framework includes three phases - inception, construction and transition and supports the entire development lifecycle from the initial concept for the product, through delivery, to operations and support and finally to retirement [23]. According to [20], in order to succeed in adopting DAD delivery teams must work intensively with enterprise architects, operations engineers, governance people, data management people, and many others. DAD teams are enterprise aware - work closely with enterprise professionals, such as enterprise architects and portfolio managers, adopt and follow enterprise guidance, leverage enterprise assets, including existing systems and data sources, enhance organizational ecosystem via refactoring enterprise assets, adopt a DevOps culture, adopt appropriate governance strategies [23].

Integrating agile methods with EA frameworks

The compliance of individual agile projects with EA frameworks is an essential issue. Combining EA frameworks with agile methods in companies is a new and challenging issue. The last ones are seen loose and extremely flexible, while frameworks such as COBIT, ITIL or TOGAF are rather considered as bureaucratic and procedural. There are several researches that have already investigated this subject.

Some authors proposed models that integrated one of the EA frameworks with Scrum. For example, [24] presented a framework that integrates the TOGAF architecture development method with Scrum. In their model the architecture vision, business architecture, information system architecture and technology architecture are developed in sprints. [25] analyzed how ITIL v.3 and agile project management can coexist in an IT organization. [26] identified risks, challenges and issues of using Scrum and COBIT.

Other papers emphasize the differences between EA frameworks and agile methods that could be considered as the main challenges of their integration [26]. EA frameworks are process-centric and designed to standardize people to the processes, while agile methods are based on people and their creativity. In EA frameworks the conformance to plan is very important and all components are extensive documented. In agile methods the change of the requirements is welcome even late in development stage and documentation must be minimal. In EA frameworks the management is characterized by command and control, while in agile team members collaborates closely and the teams are characterized by self-organization. Also, instead of analyzing risk and uncertainty thoroughly as EA framework, in the case of agile methods they are tackled empirically [27].

Despite these differences, the two approaches share the same objective: to provide business value to its customers and users. According to [28], if it is built on the strengths of both approaches, it can create enterprises "that move as a united whole without a central, commandand-control management that stifles local development and innovation". Previous presented frameworks for large-scale agile move in this direction. Moreover, both EA and agile frameworks are still IT-centric. Business architecture is the most important part of enterprises that are entirely agile.

\section{Using hybrid methods}

The Waterfall model was the dominant software development approach in large companies but, in the last years, as agile approach has grown in favor, they are increasingly transitioning from Waterfall model to Agile methodology. Each of the two software development approaches has its strengths and weak- 
nesses and fits into their own project characteristics. Some companies tried to use both of them, side by side, either in concurrent projects, or as an intermediate stage of migration from plan-driven to agile methods. However, their coexistence in the same organization was generally seen as problematic, causing tensions on all organizational levels [29] as agile methods bring drastic changes regarding team hierarchies, organizational structures, planning or controlling processes [30].

The use of hybrid control mechanism might be a solution for organizations with traditional software development routines that seek to apply agile methodology [31] and use both approaches in parallel. The hybrid mechanism is unlike traditional outcome control in the Waterfall model and also unlike emergent control specific to agile approach.

Therefore, many authors suggest that hybrid methods, by combining the plan-driven and agile methods, could represent a solution for applying agile in large organizations seeking to improve software quality and product functionality [2], [7], [32]. The mixing of agile practices with plan-driven methods might enable software development teams to take advantage of some benefits of agile development, such as adaptability to changing requirements and uncertainty, without abandoning the stability provided by the traditional methods [9]. Some companies introduced hybrid methods in order to mitigate the level of change and retain some established processes [30].

A way to create a hybrid method is to alternate the steps from the Waterfall model with steps from agile methods, like in Water-Scrum-Fall model [30]. In this hybrid plan-driven/agile approach the Waterfall model is used to define upfront work, i.e. to define essential requirements and the overall budget, or to determine the process structure and timeframe. Afterwards, Scrum is used for implementation step, during which time many Sprints are conducted until an acceptable solution is implemented. The final steps of the development process, such as software testing and delivery are again carried out using Waterfall model.
Even though hybrid plan-driven/agile methodologies have been increasingly adopted by large organizations, they were hardly investigated in literature. Moreover, related studies considered hybrid methodologies only as an intermediate stage in the migration process of large organizations from Waterfall model to agile methods [30] [31]. Therefore, very little it is known about how successful such methods are, which critical success factors ought to be considered, and which challenges might face large organizations.

Several papers tried to provide answers to such questions. For example, [9] provides a framework for determining whether a hybrid approach is appropriate for an organization or a project by evaluating the project size, volatility and interdependencies. They concluded that when software projects have high uncertainty and reciprocal interdependencies then a hybrid methodology should be implemented. In this way, hybrid methods allow large organizations to enjoy the benefits of agile development in areas where purely agile methods are not feasible. Another solution, proposed by [32], is that each organization/team should tailor a hybrid method by determining the best balance between the agile and plandriven disciplines based on a risk analysis of project characteristics versus a given method characteristics [32]. Also, they affirm that hybrid approaches are feasible and necessary for projects that combine a mix of agile and plandriven characteristics. The hybrid methodologies also present some acceptance barriers such as restricted individual autonomy and increased process complexity, and some potential weaknesses in applying agile principles like self-organizing team and flat hierarchies [30].

In the next section we will analyze all the three solutions in relation to the aforementioned challenges in applying agile in organizational context.

\section{Discussion}

In this section, first we present our general observations on the relevance of organizational context topic in the large-scale agile literature. 
Then, a discussion on the answers of our research questions follows. Next, we analyze how the solutions presented in the previous section could be used to mitigate the challenges identified in the literature.

In our opinion, two different dimensions must be taken into consideration when analyzing agile implementation at-large scale: individual level, i.e. project or team, and organizational level, that is applying agile approach within whole organization. This distinction is important because the challenges and solutions of applying agile at large-scale are different. Also, this idea highlights the need for concern about the creation of agility beyond individual team/project level in the context of the increasingly widespread use of agile approach in large organizations. Specifically, we call, through our article, for the need to investigate the coherence and concurrence between the agility of the individual projects and the whole company context.

Our literature review shows that the most researches do not make distinction between the two dimensions. Most papers deal with the topic either on individual dimension or they are not very specific. Moreover, the researches that deal explicitly with applying agile in large organization often overlook the organizational context and only several papers explicitly used an organizational perspective [5], [6], [7]. This layout increases the difficulty of making a clear classification of the existing streams of research.

Based on our literature review findings we identified 12 challenges relevant for applying large-scale agile at organizational level, which cover the RQ1. They were grouped into three categories in order to clarify the impact on large-scale agile beyond individual team/project. Working in multi-team/project environment is probably the most common challenge category cited in the literature which we found relevant for organizational context. In the same category we find the two most prominent and cited challenges for applying agile in large organizations, respectively coordinating the work of several agile teams and implementing self-organizing principle.

The second category comprises the challenges related to the organization change/transformation process, a premise for a successful implementation of agile approach in whole organization. Here, the most relevant challenges were cultural change in organization management, the integration of non-development functions, such as marketing or human resources management, into an agile environment, and the integration of agile principles and practices to the existing traditional development process. In the last category we have included the challenges regarding the alignment of individual projects to the enterprise overall business goals, with the integration of agile methods and the enterprise architecture frameworks being the most important challenge.

The literature on the large-scale agile implementation reveals three categories of solutions that address specific challenges: adapting the development environment to the agile values, principles and practices, using hybrid methods by combining agile practices with traditional, plan-driven methods, and tailoring agile methods by adjusting them to the large-scale settings. Starting from this categorization, we identified three general solutions that could be used to address the challenges of applying agile in an organizational context: the adoption of large-scale agile frameworks, the use of hybrid agile/traditional methods and the integration of agile methods with EA frameworks.

Large-scale agile frameworks are predominantly addressing the multi-team/multi project challenges category. They offer roles, artifacts and techniques that allow software development to work in more than 8 teams, such as LeSS Huge. More specifically, Nexus framework handles the specific problems of multiteams working environment by introducing an integration team that is focused on facilitating the dependencies and integration issues between teams. Also, these frameworks could be applied in order to mitigate the challenges included in our third category - alignment of individual projects to enterprise business goals. A DAD team is enterprise aware by the fact that it adopts and follows enterprise guidance, enhance organizational ecosystem via refac- 
toring enterprise assets, and adopts appropriate governance strategies [23]. Despite the opportunities offered, the application of these frameworks involves some unclear aspects that need to be investigated in the future. They refer to the context and specific conditions of organizations for their successfully application, the choice between different large-scale frameworks, how successful such methods are, and which challenges might face large organizations.

The second solution mentioned above is to use hybrid methods. Some companies are using such methods in order to mix the advantages specific both, Waterfall and agile approaches, but the most of them are considering them only as an intermediate stage in the migration process from Waterfall model to agile methods. The literature review shows that the coexistence of traditional and agile methods in the same organization is a challenge in itself and is generally seen as problematic as the two approaches are so different in terms of team hierarchies, organizational structures and planning processes.

One of the most important obstacles in using hybrid methods regards the implementation of self-organizing team principle, one of the fundamental assumptions in agile approach. This principle is not easy transferable to hybrid approach if it makes use of traditional, hierarchical management and strict controlling processes [30]. All these shortcomings create the feeling that the use of hybrid methods is rather a challenge, not a solution to apply agile in large organizations, especially due to the fact that they were hardly investigated by the researchers. Although the literature proposes some mechanisms to determine whether the hybrid approach is appropriate for a specific organization or project, or to find the best balance between the agile practices and waterfall processes, there remain issues to be investigated in the future, such as the critical success factors and acceptance barriers, the implementation of self-organizing principles in hybrid methods, the tailoring frameworks.

The third solution implies the integration of EA frameworks and agile methods. The most of large enterprises use some EA frameworks, such as COBIT, ITIL, and TOGAF in the regard of ensuring the alignment of IT with overall business goals. EA is a well-defined practice for conducting enterprise analysis, design, planning, and implementation using a holistic approach in order to guide organizations through the business, information, process, and technology changes, necessary to execute their strategies. But their holistic approach is conflicting with individual propensity of agile methods and that creates new challenges for applying agile in large organizations. Ensuring the compliance of individual agile projects within the holistic EA view is an essential aspect of architecture governance and for this reason the organizations must find the best way to approach EA capability for supporting agile development.

The literature presents some attempts to integrate an EA framework with an agile method or project management principles [24], [25], [26]. Another approach involves customizing the agile approach and practices before applying them in large organizations. Also, in order to integrate EA frameworks with agile methods, architects can start documenting standards and guidelines in collaboration with the various agile teams to create a catalogue of standards that all teams have to use [11]. Moreover, the existence of such catalogue might help to mitigate the challenges specific to the multi-team challenges category. Despite these achievements, the research on the application of agile practices and principles within EA approach is still limited.

\section{Conclusions}

Large-scale agile development received widespread interest in research and practice, although the initial fundamental assumptions suggested that agile approach is best suited to small projects and single, small development teams. A classification of the existing research in the large-scale agile field is difficult due to the lack of general agreement to the terms used in agile at large-scale area. Our literature analysis reveals that we could take into consideration two different research approaches: individual level, like project, software or team, and organization level. In our paper we 
placed at organization level with the objective to find out the challenges and solutions that are relevant for enterprise-wide agile implementation in large organizations. More specifically, we raise the need to investigate the coherence and concurrence between the agility of the individual projects and the organizational context.

Based on the literature review, we have identified 12 challenges and grouped them into three main categories: multi-team/multi-project environment (implementing self-organizing teams, coordination of several agile teams, different interpretations of agile between teams, managing and sharing knowledge with stakeholders), organizational transformation/change (management change, culture and leadership behavior change, specialized knowledge in silos, integrating non-development functions, integration of agile projects with the project environment's existing processes) and alignment of individual projects to enterprise business goals (conflicts between agile projects and holistic enterprise architecture, integration of EA frameworks and agile development, balancing between the agility of individual projects and the organization agility). The most cited challenge in literature seems to be team's coordination and the most problematic agile characteristic when applying agile in large organizations is self-organizing team.

Addressing our second research question, we have identified and analyzed three solutions for applying agile at organizational level: applying agile frameworks for large-scale, integration of agile methods with the EA frameworks and the use of hybrid agile/traditional methods.

Our paper can be used by academics as a roadmap for future research and by practitioners to understand and address the challenges and solutions of agile methods adoption at organizational level.

\section{References}

[1] M. Jørgensen, "Do agile methods work for large software projects?," in Proc. The International Conference on Agile Software Development, Porto, Portugal,
2018, pp. 179-190.

[2] T. Dreesen and P. Diegmann, "Journey Towards Agility: Where Are We Now And Where Are We Heading?," in Proc. The International Conference on Information Systems, Seoul, South Korea, 2017, pp. 1-11.

[3] F. Dumitriu, G. Meșniță, D. Oprea and L. Radu, "The Need for a Framework in Analyzing the Applying Agile at LargeScale," in Proc. The 17th International Conference on Informatics in Economy, Iași, Romania, 2017, pp. 417-424.

[4] K. Rolland, T. Dingsoyr, B. Fitzgerald and K. Stol, "Problematizing agile in the large: alternative assumptions for largescale agile development," in Proc.The 39th International Conference on Information Systems, Dublin, Irland, 2016, pp. $1-20$.

[5] R. Wendler, "Dimensions of Organizational Agility in the Software and IT Service Industry: Insights from an Empirical Investigation," Communications of the Association for Information Systems, vol. 39, no. 1, paper 21, 2016.

[6] M. Paasivaara and C. Lassenius, "Challenges and success factors for large-scale agile transformations: A research proposal and a pilot study," in Proc. The Scientific Workshop Proceedings of XP2016, 2016, article no. 9.

[7] M.F. Abrar, S. Rehman, N. Rashid and S. Ali, "Large Scale Agile Adoption Model from Management Perspective," International Journal of Computer Applications, vol. 152, no. 2, pp. 31-35, 2016.

[8] T. Karvonen, H. Sharp and L. Barroca, "Enterprise Agility: Why Is Transformation so Hard?," in Proc. The 19th International Conference on Agile Processes in Software Engineering and Extreme Programming, Porto, Portugal, 2018, pp. 131-145.

[9] M. Lindvall, D. Muthig, A. Dagnino, C. Wallin, M. Stupperich, D. Kiefer, J. May and T. Kahkonen, "Agile software development in large organizations,"Computer, vol. 37, no. 12, pp. 26-34, 2004. 
[10] J. Barlow, J. Giboney, M. Keith, D. Wilson, R. Schuetzler, P. Lowry and A. Vance, "Overview and guidance on agile development in large organizations,"Communications of the Association for Information Systems, vol. 29, no. 2, pp. 25-44, 2011.

[11] B. Boehm and R. Turner, "Management challenges to implementing agile processes in traditional development organizations,"IEEE Software, vol. 22, pp. 3039, 2005.

[12] Ö. Uludag, M. Kleehaus, C. Caprano and F. Matthes, "Identifying and Structuring Challenges in Large-Scale Agile Development Based on a Structured Literature Review," in Proc. The 22nd International Conference on Enterprise Distributed Object Computing, Stockholm, Sweden, 2018, pp 191-197.

[13] B. Hobbs and Y. Petit, "Agile Methods on Large Projects in Large Organizations," Project Management Journal, vol. 48, no. 3, pp. 3-19, 2017.

[14] T. Dingsøyr and D. P. K. Falessi, “Agile Development at Scale: The Next Frontier,'IEEE Software, vol. 36, no. 2, pp. 30-38, 2019.

[15] R. Duijs, P. Ravesteyn and S. Marlies, "Adaptation of enterprise architecture efforts to an agile environment," Proc. The 31ST Bled eConference Digital Transformation: Meeting the Challenges, Blend, Slovenia, 2018, pp. 389-400.

[16] A. Gill, "Adaptive enterprise architecture driven agile development," in Proc.The International Conference on Information Systems Development, Harbin, China, 2015, pp. 1-9.

[17] J. Persson, J. Nørbjerg and P. Nielsen, "Improving ISD Agility in Fast-Moving Software Organizations," in Proc. The European Conference on Information Systems, Istanbul, Turkey, 2016.

[18] K. ConboyandN. Carroll. "Implementing Large-Scale Agile Frameworks: Challenges and Recommendations, "IEEE Software, vol. 36, no. 2, pp. 44-50, 2019.

[19] Scaled Agile, Inc. (2017). SAFe® 4.5 Introduction-Overview of the Scaled Agile
Framework® for Lean Enterprises https://www.scaledagileframework.com/

[20] M. AlqudahandR. Razali. "A review of scaling agile methods in large software development,"International Journal on Advanced Science, Engineering and Information Technology, vol. 6, no. 6, pp. 828-837, 2016.

[21] K. Schwaber. (2018). Nexus ${ }^{\mathrm{TM}}$ Guide. The Definitive Guide to scaling Scrum with Nexus: The Rules of the Game https://www.scrum.org/resources/nexusguide.

[22] Smartsheet Inc. 2019. Scaling Agile for Enterprises: A Comparison of SAFe Agile, Nexus, Disciplined Agile 2.0 (DAD), and Large-Scale Scrum (LeSS), https://www.smartsheet.com/scaling-agile-for-enterprises-a-comparison-of-safeagile-nexus-disciplined-agile-dad-andlarge-scale-scrum.

[23] S.W. Ambler andM. Lines. "The disciplined agile process decision framework,"in Proc.The International Conference on Software Quality, Vienna, Austria, 2016, pp. 3-14.

[24] S. Hanschke, J. Ernsting andH. Kuchen. "Integrating agile software development and enterprise architecture management," in Proc. The 48th Hawaii International Conference on System Sciences, Hawaii, USA, 2015, pp. 4099-4108.

[25] B. Verlaine, I.JuretaandS. Faulkner. "How Can ITIL and Agile Project Management Coexist?," in Proc. The International Conference on Exploring Services Science, Bucharest, Romania, 2016, pp. 327-342.

[26] N. Ozkan. "Risks, challenges and issues in a possible SCRUM and COBIT marriage, "in Proc. The Asia-Pacific Software Engineering Conference, New Delhi, India, 2015, pp. 111-118.

[27] O. Ktata andG. Lévesque. "Agile development: Issues and avenues requiring a substantial enhancement of the business perspective in large projects," Proc. The 2nd Canadian Conference on Computer Science and Software Engineering, Quebec, Canada, 2009, pp. 59-66. 
[28] M. Lankhorst. "Enterprise Architecture and Agile Development: Opposites Attract?,"https://bizzdesign.com/blog/enterprise-architecture-and-agile-development-opposites-attract/.

[29] K. Dikert,M. Paasivaara and C. Lassenius. "Challenges and Success Factors for Large-Scale Agile Transformations: A Systematic Literature Review," Journal of Systems and Software, vol. 119, pp. 87-108.

[30] S. Schlauderer, S. Overhage and B. Fehrenbach, B. "Widely used but also highly valued? Acceptance factors and their perceptions in water-scrum-fall projects," in
Proc. The International Conference on Information Systems, Fort Worth, Texas, 2015.

[31] L. Mahadevan, W.J. Kettinger and T.O. Meservy. "Running on Hybrid: Control Changes when Introducing an Agile Methodology in a Traditional "Waterfall" System Development Environment," Communications of the Association for Information Systems, vol. 36, articol 5, pp. 76-102, 2015.

[32] B. Boehm, "Get ready for agile methods, with care," Computer, vol. 1, no. 35, pp. 64-69, 2002.

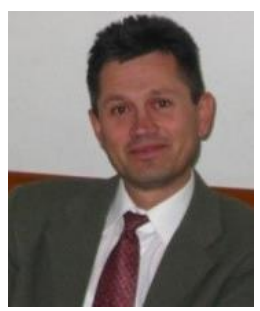

Florin DUMITRIU is $\mathrm{PhD}$ Professor at the Alexandru Ioan Cuza University of Iaşi, Faculty of Economics and Business Administration. He is currently teaching and researching subjects, such as Systems Analysis and Design, Human Resource Information Systems, Global Software Development, Information Systems Offshoring. He is the author and co-author of 8 books on systems analysis and design and business information systems and 21 scientific papers published in indexed journals and conference proceedings.

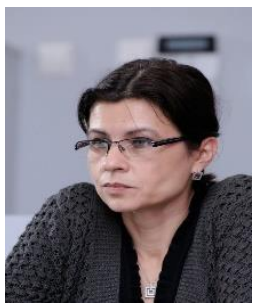

Gabriela MESNITA is PhD professor of Business Information Systems at "Alexandru Ioan Cuza" University of Iasi, Faculty of Economics and Business Administration. She received her PhD in Business Information Systems in 1999. Currently professor Mesnita is $\mathrm{PhD}$ adviser. She published as author or coauthor over 20 books and university courses or chapters. Also she presented and/or published over 80 articles in Romania, Poland, China, Turkey, Italy etc. Her research interests include information systems (analysis and design, outsourcing); e-business; project management; IoT.

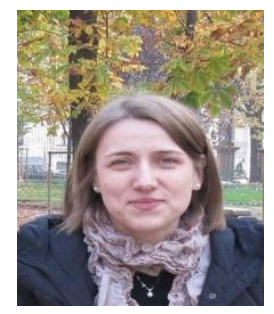

Laura-Diana V. RADU (b. September 26, 1978) received his BSc in Accounting and Information Systems (2001), M.Sc. in Business Information Systems (2003), PhD in Accounting (2006) from "Alexandru Ioan Cuza" University of Iasi. Since 2009, she was a researcher with the Research Department, Faculty of Economics and Business Administration, Alexandru Ioan Cuza University of Iasi. Currently she is a lecturer in the Department of Business Information Systems from Faculty of Economics and Business Administration. She is the author of one book, 10 chapters and more than 66 articles. Her research interests include accounting information systems, green information and communication technology, green information systems, smart cities and agile project management. 